\title{
Posterior reversible encephalopathy syndrome (PRES) of the brainstem
}

\author{
Yosuke Hayashi $^{1}$, Tomoaki Hashida ${ }^{1}$, Megumi Yazaki ${ }^{1}$, Tomohiko Uchida ${ }^{1}$, and Eizo \\ Watanabe $^{1}$ \\ ${ }^{1}$ Eastern Chiba Medical Center
}

January 4, 2022

\begin{abstract}
A 71-year-old man had disordered consciousness whose Glasgow Coma Scale was E4V1M5. His blood pressure was high, but there was no abnormality in the cerebrospinal fluid examination. The MRI finding reveals a high-intensity area at the pons without the blood flow interruption. Thus, he has diagnosed with brainstem PRES

Clinical Image

Posterior reversible encephalopathy syndrome (PRES) of the brainstem

Yosuke Hayashi $^{1}$, Tomoaki Hashida ${ }^{1,2}$, Megumi Yazaki ${ }^{1}$, Tomohiko Uchida ${ }^{1,3}$, Eizo Watanabe ${ }^{1,2}$

${ }^{1}$ Department of Emergency and Critical Care Medicine, Eastern Chiba Medical Center

${ }^{2}$ Department of General Medical Science, Chiba University Graduate School of Medicine

${ }^{3}$ Department of Neurology, Eastern Chiba Medical Center

Short running title: PRES of the brainstem

Corresponding author: Eizo Watanabe

Department of Emergency and Critical Care Medicine, Eastern Chiba Medical Center, 3-6-2 Okayamadai, Togane City, Chiba 283-8686, Japan

Tell: +81-475-50-1199

Email: eizo-watanabe@umin.ac.jp
\end{abstract}

\section{CASE REPORT}

A 71-year-old man was taken to our hospital because of disordered consciousness. His conscious level was E4V1M5 with Glasgow Coma Scale on admission. His blood pressure was 209/124mmHg, but there was no abnormality in both laboratory testing and cerebrospinal fluid examination. The MRI finding reveals a high-intensity area at the pons with FLAIR Imaging and ADC map (Fig. 1A and 1B ), but isointensity of DWI and no evidence of the blood flow interruption. Thus, the patient was diagnosed with PRES of the brainstem. Although his consciousness worsened on the following day to E2V1M5, it recovered on the 9th day with antihypertensive treatments. The patient was discharged on the 20th day without any sequelae.

\section{DISCUSSION}

PRES refers to a disorder of reversible subcortical vasogenic brain edema in patients with acute neurological symptoms. The pathophysiology is mainly hyperperfusion of the brain as a sequel of hypertension. This is 
suggested to be associated with hyperpermeability of cerebral blood vessels[1]. The typical MRI finding is the symmetric vasogenic edema in the parietal and occipital lobes. However, up to the $27 \%$ of cases occur at the brainstem[2]. It is, therefore, crucial to bear PRES in mind even though the clinical imaging is atypical.

\section{DISCLOSURE}

* Approval of the research protocol: N/A

* Informed Consent: Written informed consent was obtained from the next of kin for the publication of any potentially identifiable images or data included in this article.

* Registry and the Registration No. of the study/Trial: N/A

* Animal Studies: N/A

* Conflict of Interest: None.

\section{REFERENCES}

1. Fugate JE, Rabinstein AA: Posterior reversible encephalopathy syndrome: clinical and radiological manifestations, pathophysiology, and outstanding questions . Lancet Neurol 2015,14 (9):914-925.

2. Fugate JE, Claassen DO, Cloft HJ, Kallmes DF, Kozak OS, Rabinstein AA: Posterior reversible encephalopathy syndrome: associated clinical and radiologic findings . Mayo Clin Proc 2010,85 (5):427-432.

A

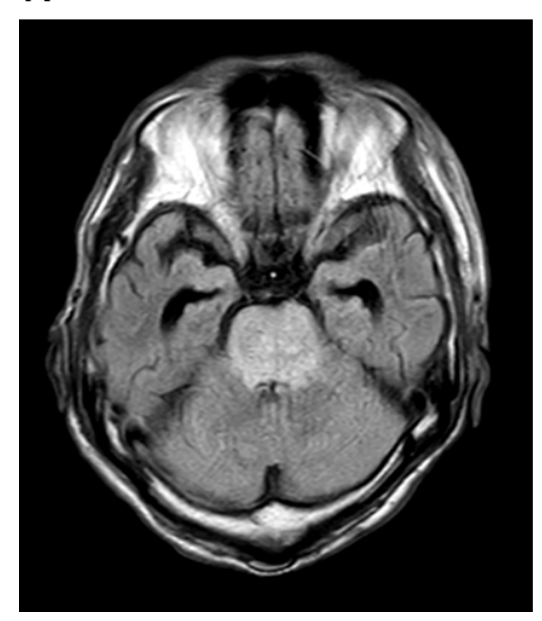

B

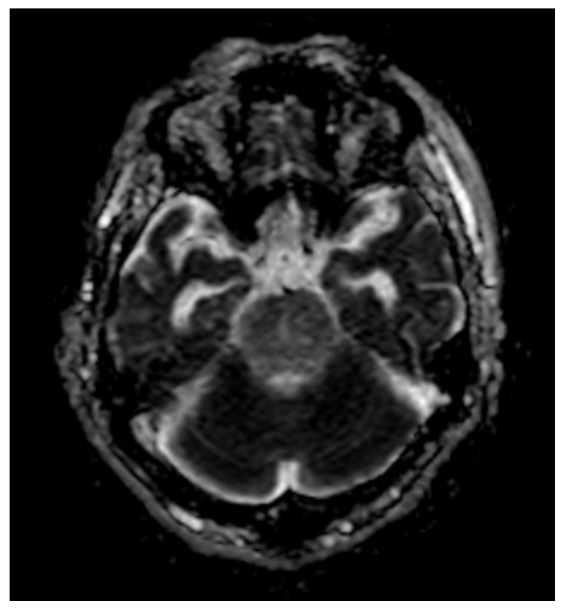

